\title{
SARS-COV-2 VIRUS MUTATION AND LOSS OF TREATMENT AND PREVENTIVE MEASURES AS WE KNOW IT NOW
}

\section{Francesco Chirico ${ }^{1,2}{ }^{\infty}$, Dorota Sagan ${ }^{3}$, Anna Markiewicz ${ }^{3}$, Julia Popieluch ${ }^{3}$, Michal Pruc ${ }^{4}\left({ }^{0}, K^{2}\right.$ arol Bielski ${ }^{4}$, Maciej Cyran ${ }^{3,4}$}

\author{
'Post-graduate School of Occupational Health, Università Cattolica del Sacro Cuore, Rome, Italy \\ ${ }^{2}$ Health Service Department, Italian State Police, Milan, Italy \\ ${ }^{3}$ Maria Sklodowska-Curie Medical Academy, Warsaw, Poland \\ ${ }^{4}$ Research Unit, Polish Society of Disaster Medicine, Warsaw, Poland
}

KEY WORDS: SARS-CoV-2, COVID-19, mutation, pandemic, prevention

Disaster Emerg Med J 2021; 6(4): 204-205

To the Editor,

in the current epidemiological situation, while the infectivity of the delta variant of the SARSCoV-2 coronavirus has become so high compared to the variants known to us, amounting to almost twice as much, there is more concern about the existing preventive measures, which are known to us in the form of vaccines and treatment of patients hospitalized in connection with a pandemic. Such a rapid spread of the virus in society will mainly affect people who have not been vaccinated so far, but indirectly there are also discussions about the effectiveness of vaccines themselves, which, although they are effective and protect against severe disease related to the new variant, their effectiveness is reduced - adjusted effectiveness measured as protection from contracting COVID-19 decreased from $91.7 \%$ to $79.8 \%$ and the adjusted efficacy measured as protection against COVID-19 hospitalization remained relatively unchanged - a decrease from $95 \%$ to $92 \%$ [1]. It should also be pointed out that the vaccination rate is inversely correlated with the mutation rate of the Delta SARS-CoV-2 variant in 16 countries $\left(R^{2}=0.878\right)$, which strongly suggests that full SARS-CoV-2 vaccination is necessary to inhibit subsequent mutations, and the number of unvaccinated people around the world is still very large and they will be responsible for the emergence of further new mutations. The SARS-CoV-2 virus mutates incredibly quickly, as exemplified by variants such as delta or lambda [2]. The mild and asymptomatic delta course, especially among vaccinated persons, where new studies report up to $68 \%$ of positive test cases in the vaccinated population, will spread the disease very rapidly in both vaccinated and unvaccinated persons, possibly leading to the emergence of new variants which may eventually prove refractory to both the vaccination and the current treatment as we know it, even though we know it ourselves. The cure for COVID-19 disease remains unknown to us so far [3]. It is imperative that the entire population be vaccinated as soon as possible, even under the condition of compulsory vaccination, as the current COVID-19 variants in the vaccinated population are passing through very gently compared to unvaccinated people. This will reduce the number of hospitalizations and mortality, and will significantly reduce the risk of mutations and the emergence of new mutations that may turn out to be insensitive to vaccinations and known drugs, which can occur very quickly, looking at the number of varieties and sub-varieties of individual variants known to us so far. 


\section{Conflict of interest}

All authors declare no conflict of interest.

\section{REFERENCES}

1. Rosenberg ES, Holtgrave DR, Dorabawila V, et al. New COVID-19 Cases and Hospitalizations Among Adults, by Vaccination Status - New York, May 3-July 25, 2021. MMWR Morb Mortal Wkly Rep. 2021;
70(37): 1306-1311, doi: 10.15585/mmwr.mm7037a7, indexed in Pubmed: 34529645.

2. Yeh TY, Contreras G. Full vaccination against COVID-19 suppresses SARSCoV-2 delta variant and spike gene mutation frequencies and generates purifying selection pressure. , doi: 10.1101/2021.08.08.21261768.

3. Riemersma K, Grogan B, Kita-Yarbro A, et al. Shedding of Infectious SARS-CoV-2 Despite Vaccination. , doi: 10.1101/2021.07.31.21261387. 